\title{
Serum soluble interleukin-2 receptor levels for screening for malignant lymphomas and differential diagnosis from other conditions
}

\author{
JUN MURAKAMI ${ }^{1}$, KOTARO ARITA ${ }^{1}$, AKINORI WADA ${ }^{1}$, HIROSHI MIHARA ${ }^{1}$, \\ HIDEKI ORIGASA ${ }^{2}$, MIKA KIGAWA ${ }^{3}$, ICHIRO YASUDA ${ }^{1}$ and TSUTOMU SATO ${ }^{1}$ \\ ${ }^{1}$ Department of Gastroenterology and Hematology, University of Toyama, Graduate School of Medicine and \\ Pharmaceutical Sciences; ${ }^{2}$ Division of Biostatistics and Clinical Epidemiology, University of Toyama, \\ Graduate School of Medicine, Toyama 930-0194; ${ }^{3}$ Faculty of Health and Welfare, \\ Kanagawa University of Human Services, Yokosuka, Kanagawa 238-8522, Japan
}

Received April 1, 2019; Accepted July 22, 2019

DOI: $10.3892 / \mathrm{mco} .2019 .1922$

\begin{abstract}
The serum soluble interleukin 2 receptor (sIL2R) level is elevated in patients with most types of lymphoid neoplasms, and is also elevated in patients with solid tumors or reactive conditions, such as severe inflammation. To evaluate the diagnostic significance of sIL2R levels for the screening and differential diagnosis of lymphomas, data from 248 consecutive adult patients with suspected lymphoma were retrospectively analyzed in order to determine its diagnostic characteristics and the clinical parameters that affect diagnosis. In 133 patients with aggressive or indolent lymphomas or related neoplasms, the sIL2R level was higher (median: $920 \mathrm{U} / \mathrm{ml}$, standard deviation: 7,312 U/ml) compared with that of 115 patients with other diagnoses (median: $520 \mathrm{U} / \mathrm{ml}$, standard deviation: $727 \mathrm{U} / \mathrm{ml}$ ), including solid tumors, infection, inflammation, and others. When the cutoff value of sIL2R was $1,104 \mathrm{U} / \mathrm{ml}$, the specificity was $80 \%$, at which point lymphoma was suspected. When the threshold levels were increased from 1,500 to $2,000 \mathrm{U} / \mathrm{ml}$, the specificity increased from 87 to $93 \%$,
\end{abstract}

Correspondence to: Dr Jun Murakami, Department of Gastroenterology and Hematology, University of Toyama, Graduate School of Medicine and Pharmaceutical Sciences, 2630 Sugitani, Toyama 930-0194, Japan

E-mail: jmura-tym@umin.ac.jp

Abbreviations: ALL, acute lymphoblastic leukemia; ATLL, adult T-cell lymphoma/leukemia; AUC, area under the curve; CLL, chronic lymphocytic leukemia; CRP, C-reactive protein; FUO, fever of unknown origin; IL-2, interleukin 2; LDH, lactate dehydrogenase; LR+, positive likelihood ratio; LR-, negative likelihood ratio; NPV, negative predictive value; PPV, positive predictive value; sIL2R, serum soluble interleukin-2 receptor; $\mathrm{Sn}$, sensitivity; $\mathrm{Sp}$, specificity; $\mathrm{ROC}$, receiver operating characteristic; $\mathrm{WBC}$, white blood cell

Key words: serum soluble interleukin-2 receptor, malignant lymphoma, screening, tumor marker, diagnostic value with the positive likelihood ratio increasing from 2.99 to 4.97 , strongly suggesting the diagnosis of lymphoma. The receiver operating characteristic curve for prediction of lymphoma by sIL2R revealed that the area under the curve was 0.695 . The curve was nearest to the left corner of the plot when the threshold was $1,946 \mathrm{U} / \mathrm{ml}$; at this point, the sensitivity, specificity and positive likelihood ratio were $35 \%, 93 \%$ and 5.06, respectively. Multivariate analysis demonstrated that an age $>46$ years and lactate dehydrogenase level $>173 \mathrm{U} / 1$ appeared to increase the risk of malignant lymphoma diagnosis. Although sIL2R appears to be a less specific marker for the screening of lymphomas, its detection at higher levels strongly suggests the diagnosis of lymphomas. Therefore, sIL2R may be more useful compared with any other parameter for lymphoma diagnosis, provided other false-positive conditions are taken into consideration.

\section{Introduction}

Interleukin 2 (IL-2), which has been identified in activated $\mathrm{T}$ cells, stimulates an immune response on target cells via a high affinity receptor composed of $\alpha, \beta$ and $\gamma$ subunits. The interaction between IL-2 and its receptors on target cells plays a pivotal role in activating and maintaining immune responses. The soluble form of the IL2R $\alpha$ subunit (CD25) appears to be released into the serum from the membranes of activated lymphocytes after shedding by proteolytic cleavage. Soluble IL-2 receptor $\alpha$ (sIL2R) is detected in the serum of healthy individuals and increases in association with various types of inflammation or neoplasms (1-3). However, the mechanism and functional significance of elevated sIL2R levels for each pathological condition remain unclear.

Serum sIL2R levels have been found to be elevated in most types of hematolymphoid neoplasms, including Hodgkin's lymphomas, non-Hodgkin lymphomas, acute lymphoblastic leukemia (ALL), chronic lymphocytic leukemia (CLL), multiple myeloma, and others (3). Moreover, the highest levels of sIL2R have been reported in adult T-cell lymphoma/leukemia (ATLL) (4) and hairy cell leukemia (5). 
In patients with hematolymphoid neoplasms, most of the serum sIL2R is derived from the neoplastic cells themselves, directly reflecting the tumor burden and disease activity; therefore, it may be considered as a true tumor marker. In certain B-cell lymphomas, proteinases derived from tumor-associated macrophages in the tumor microenvironment also appear to play an important role in producing sIL2R (6). In several non-lymphoid solid tumors, serum sIL2R levels are significantly higher compared with those in healthy individuals, although this increase is not as notable as that observed in hematolymphoid neoplasms $(1,7,8)$. In non-lymphoid solid tumors, increased serum sIL2R levels may originate from tumor cells, as well as from activated lymphoid cells, circulating mononuclear cells, or tumor-infiltrating lymphocytes (3). Furthermore, elevated sIL2R levels have been detected in a growing number of pathological conditions, including infections, autoimmune or other inflammatory diseases, allograft rejection, graft-vs.-host disease after allogeneic hematopoietic stem cell transplantation, and hemophagocytic lymphohistiocytosis (3).

Although the mechanisms and clinical significance of elevated sIL2R levels are unclear, the clinical usefulness of sIL2R has been evaluated for the diagnosis, staging, prognosis and post-treatment monitoring in lymphomas or other diseases (3). However, the availability of data on the significance of sIL2R levels in the differential diagnosis of lymphoma from other diseases in the clinical setting is limited $(1,7,8)$. In the present study, patients with suspected lymphoma were retrospectively analyzed and the sIL2R levels were measured, the final diagnoses were compared, and then the diagnostic characteristics and clinical parameters that affect the diagnostic value were evaluated.

\section{Patients and methods}

Ethics approval. The present study was approved by the Ethics Committee of the University of Toyama. The use of an opt-out method enabled refusal to participate in disclosure documents.

Patient population. A total of 248 consecutive adult patients (aged $\geq 18$ years) who had suspected malignant lymphoma and had their serum sIL2R levels measured by attending physicians in Toyama University Hospital between January 2004 and December 2007 were retrospectively analyzed.

Data collection. Data on clinical parameters, including age, sex, white blood cell (WBC) count, C-reactive protein (CRP) levels (normal range: 0.00-0.14 mg/dl), serum lactate dehydrogenase (LDH) levels (normal range: 124-222 U/l) and serum sIL2R levels, were extracted from the medical record database. Clinical symptoms at presentation, initial differential diagnosis and final diagnosis were reviewed in the medical records for each patient. Standard histological diagnosis of lymphoma by hematopathologists was based on the World Health Organization 2008 classification.

sIL2R measurement. The serum sIL2R levels were measured with a sandwich-enzyme immunoassay (IL-2R test; BML; cat. no. 221ADAMX00007000). The normal range was defined as 122-496 U/ml following the manufacturer's information.
Statistical analyses. The sensitivity $(\mathrm{Sn})$ and specificity (Sp) of the sIL2R levels for the diagnosis of lymphoma and other lymphoid neoplasms were evaluated, and the positive and negative predictive values were also determined. A receiver operating characteristic (ROC) curve was used to determine the diagnostic accuracy and cutoff value of sIL2R. Differences between the two groups were evaluated using the Mann-Whitney U test. Comparisons among multiple groups were made using the Kruskal-Wallis test, and post hoc group comparisons were performed with the Steel-Dwass test. A multivariate logistic regression model was used to determine risk factors for lymphomas. All data were considered statistically significant if the P-values were $<0.05$. The analyses were performed using EZR version 2.4-0 (Saitama Medical Center, Jichi Medical University), which is a graphical user interface for R (The R Foundation for Statistical Computing) (9).

\section{Results}

Patient characteristics. A total of 248 patients with lymphoma suspected by attending physicians based on clinical, laboratory or imaging findings were included in the present study. Lymphoma was suspected due to varying reasons. The common presentations and differential diagnoses for lymphomas included solid tumors with uncommon presentation, inflammatory symptoms, liver dysfunction, neurological symptoms of unexplained etiology, or even unexplained complaints. The final diagnosis of lymphoma subtype or differential diagnoses were established. The patient characteristics are summarized in Table I. Of the 248 patients, 97 were diagnosed with aggressive lymphomas, 23 with indolent lymphomas, 8 with plasma cell disorders including plasma cell myeloma, and 5 with CLL. The remaining 115 patients were diagnosed with neoplasms other than lymphoid. The most common diagnosis was non-lymphoid solid tumor $(n=50)$, followed by infection $(n=32)$, non-infectious inflammatory disorders (autoimmune diseases or allergy; $n=18$ ) and miscellaneous $(\mathrm{n}=15)$.

The histological subtypes of aggressive or indolent lymphomas and other lymphoid neoplasms are listed in Table II. Among patients diagnosed with non-lymphoid solid neoplasms, the most common types of tumors were of gastrointestinal or urogenital origin, followed by primary unknown carcinomas, non-epithelial tumors, abdominal lymph node enlargement, and splenic or bone tumors without histological confirmation.

Comparison of each parameter in patients with lymphoid tumors or other diagnoses. The sIL2R levels and other parameters were compared between the two groups: Malignant lymphomas, including CLL and plasma cell disorders (ML group) and other diagnoses (other group) (Table III). The median age of the patients in the ML group was significantly higher compared with that in the other group (67 years vs. 60 years, respectively; $\mathrm{P}=0.02$ ). The median WBC count in the ML group was similar to that of the other group $(4,570 / \mu 1$ vs. $4,680 / \mu 1)$. The median serum CRP level in the ML group was somewhat higher compared with that of the other group, but the difference was not statistically significant 
Table I. Patient characteristics.

\begin{tabular}{|c|c|c|c|c|}
\hline Diagnosis & $\mathrm{n}$ & $\mathrm{M} / \mathrm{F}$ & Mean age (range), years & Fever $(+/-)$ \\
\hline Lymphomas & 120 & $71 / 49$ & $64.1(21-90)$ & $16 / 104$ \\
\hline Aggressive & 97 & $62 / 35$ & $64.6(21-90)$ & $15 / 82$ \\
\hline Indolent & 23 & $9 / 14$ & $61.8(32-82)$ & $1 / 22$ \\
\hline \multicolumn{5}{|l|}{ Other lymphoid neoplasms } \\
\hline Plasma cell disorders & 8 & $4 / 4$ & $62.3(48-70)$ & $0 / 8$ \\
\hline CLL & 5 & $1 / 4$ & $70.4(54-83)$ & $0 / 5$ \\
\hline Non-hematolymphoid tumors & 50 & $27 / 23$ & $65.4(19-87)$ & $5 / 45$ \\
\hline Histologically proven & 43 & $22 / 21$ & $65.0(19-87)$ & $5 / 38$ \\
\hline Not histologically proven & 7 & $5 / 2$ & $68.0(49-79)$ & $0 / 7$ \\
\hline Infection & 32 & $12 / 20$ & $43.0(20-78)$ & $14 / 18$ \\
\hline Lymphadenopathy & 23 & $9 / 14$ & $38.0(20-76)$ & $10 / 13$ \\
\hline Gastrointestinal & 7 & $2 / 5$ & $55.0(27-78)$ & $2 / 5$ \\
\hline Others & 2 & $1 / 1$ & $63.0(56-70)$ & $2 / 0$ \\
\hline Non-infectious inflammation & 18 & $11 / 7$ & $54.1(24-80)$ & $5 / 13$ \\
\hline Autoimmune & 10 & $6 / 4$ & $61.8(28-80)$ & $2 / 8$ \\
\hline Allergy & 3 & $1 / 2$ & $47.7(24-71)$ & $2 / 1$ \\
\hline Others & 5 & $4 / 1$ & $60.6(28-80)$ & $1 / 4$ \\
\hline Miscellaneous & 15 & $7 / 8$ & $65.1(35-85)$ & $1 / 14$ \\
\hline
\end{tabular}

CLL, chronic lymphocytic leukemia; M, male; F, female.

Table II. Histological diagnosis of patients with lymphoid neoplasms.

\begin{tabular}{|c|c|c|c|}
\hline Category & Diagnosis & $\mathrm{M} / \mathrm{F}$ & Mean age (range), years \\
\hline \multirow[t]{10}{*}{ Aggressive lymphomas } & Diffuse large B-cell lymphoma & $41 / 27$ & $66(24-90)$ \\
\hline & Intravascular large B-cell lymphoma & $1 / 2$ & $66(69-76)$ \\
\hline & Mantle cell lymphoma & $4 / 1$ & $72(46-83)$ \\
\hline & Follicular lymphoma, grade $3 \mathrm{~A}$ & $3 / 1$ & $62(54-81)$ \\
\hline & Classical Hodgkin's lymphomas & $4 / 0$ & $46(21-59)$ \\
\hline & Peripheral T-cell lymphoma & $2 / 0$ & $57(50-64)$ \\
\hline & Angioimmunoblastic T-cell lymphoma & $3 / 1$ & $77(71-79)$ \\
\hline & ATLL & $0 / 1$ & 60 \\
\hline & NK/T-cell lymphoma & $4 / 0$ & $49(26-71)$ \\
\hline & Methotrexate-related LPD & $0 / 2$ & $58(55-61)$ \\
\hline \multirow[t]{5}{*}{ Indolent lymphomas } & Follicular lymphoma, grade 1-2 & $3 / 5$ & $62(43-77)$ \\
\hline & MALT type lymphoma & $3 / 9$ & $63(32-82)$ \\
\hline & Nodal marginal zone B-cell lymphoma & $1 / 0$ & 32 \\
\hline & Lymphoplasmacytic lymphoma & $1 / 0$ & 62 \\
\hline & Cutaneous T-cell lymphoma & $1 / 0$ & 68 \\
\hline CLL & B-CLL & $1 / 4$ & $70(54-83)$ \\
\hline \multirow[t]{6}{*}{ Plasma cell disorders } & Multiple myeloma & $1 / 1$ & $64(59-68)$ \\
\hline & Plasmacytoma & $1 / 0$ & 56 \\
\hline & POEMS syndrome ${ }^{a}$ & $0 / 1$ & 48 \\
\hline & AL-amyloidosis & $1 / 0$ & 70 \\
\hline & Light chain deposition disease & $0 / 1$ & 70 \\
\hline & IgM-MGUS & $1 / 1$ & $63.5(51-76)$ \\
\hline
\end{tabular}

aPolyneuropathy, organomegaly, endocrinopathy, monoclonal gammopathy and skin changes. NK, natural killer; LPD, lymphoproliferative disease; MALT, mucosa-associated lymphoid tissue; CLL, chronic lymphocytic leukemia; MGUS, monoclonal gammopathy of undetermined significance; ATLL, adult T-cell lymphoma/leukemia; M, male; F, female. 
Table III. Comparison of characteristics between the ML and other groups.

\begin{tabular}{|c|c|c|c|c|c|}
\hline & \multicolumn{2}{|c|}{ ML group } & \multicolumn{2}{|c|}{ Other group } & \multirow[b]{2}{*}{ P-value } \\
\hline & Median (range) & Mean \pm SD & Median (range) & Mean \pm SD & \\
\hline Age, years & $67(21-90)$ & $64.2 \pm 13.3$ & $60(19-87)$ & $57.4 \pm 18.9$ & 0.02 \\
\hline WBC count (cells/ $\mu 1)$ & $4,570(1,950-84,590)$ & $8,136 \pm 9,745$ & $4,680(1,920-17,470)$ & $6,753 \pm 3,008$ & 0.89 \\
\hline C-reactive protein $(\mathrm{mg} / \mathrm{dl})$ & $0.40(0-17.3)$ & $2.12 \pm 3.59$ & $0.20(0-27.1)$ & $1.99 \pm 4.40$ & 0.07 \\
\hline Lactate dehydrogenase (U/l) & $217(97-1,269)$ & $295 \pm 219$ & $182(99-4435)$ & $308 \pm 565$ & $<0.01$ \\
\hline sIL2R (U/ml) & $920(159-58,089)$ & $3,240 \pm 7,312$ & $520(150-4,433)$ & $786 \pm 727$ & $<0.01$ \\
\hline
\end{tabular}

ML, malignant lymphoma; SD, standard deviation; WBC, white blood cell; sIL2R, serum soluble interleukin-2 receptor.

(0.4 mg/dl vs. $0.2 \mathrm{mg} / \mathrm{dl}$, respectively; $\mathrm{P}=0.066)$. The median LDH level in the ML group was significantly higher compared with that in the other group (217 U/1 vs. $182 \mathrm{U} / 1$, respectively; $\mathrm{P}<0.01)$. The median serum sIL2R level in the ML group was significantly higher compared with that in the other group $(920 \mathrm{U} / \mathrm{ml}$ vs. $520 \mathrm{U} / \mathrm{ml}$, respectively; $\mathrm{P}<0.001)$. Similar results were observed if patients with CLL and plasma cell disorders were extracted from the lymphoma group (data not shown).

sIL2R levels in each category and subcategory. The comparison between serum sIL2R levels in the ML and the other groups is presented in Fig. 1A. The serum sIL2R levels in each of the disease categories are shown in Fig. 1B. Although aggressive lymphomas were associated with the highest sIL2R levels, indolent types of lymphomas appeared to have sIL2R levels similar to those of other disease categories, including non-hematological tumors and infection, among others.

The ML group was subcategorized into aggressive or indolent histological types, CLL and plasma cell disorders, including plasma cell myeloma. Aggressive lymphomas exhibited the highest sIL2R levels, followed by indolent lymphomas, CLL and plasma cell disorders (Fig. 1C). One patient with ATLL (acute type) had the highest levels of sIL2R $(58,089 \mathrm{U} / \mathrm{ml})$. The sIL2R level in patients with aggressive T/NK lymphomas except ATLL (median, 5,080 U/ml; range, 556-15,087 U/ml) was significantly higher $(\mathrm{P}<0.002)$ compared with that in patients with aggressive $\mathrm{B}$-cell lymphomas (median, 1,046 U/ml; range, 257-17,709 U/ml).

The sIL2R levels in various non-lymphoid solid tumors are shown in Fig. 1D. Of note, 2 of 3 patients with extremely high sIL2R levels $(>2,000 \mathrm{U} / \mathrm{ml})$ had advanced tumors with splenic involvement. The sIL2R levels in various infectious diseases are shown in Fig. 1E. Mild or moderate increases in sIL2R levels were observed. The sIL2R levels in various inflammatory diseases are shown in Fig. 1F. Of note, 3 of 4 patients with sIL2R levels $>2,000 \mathrm{U} / \mathrm{ml}$ had a drug allergy or another IgG4-related disease (Mikulicz disease).

Chronological increase of $S I L 2 R$ levels in relation to disease progression. In our cohort, 13 patients with diffuse large B-cell lymphoma had their sIL2R levels re-measured prior to treatment (interval range, 19-168 days; median, 27 days). The evaluation of sIL2R levels (median, 2,685 U/ml) revealed that they had increased from the initial measurement (median, 1,156 U/ml), consistently with disease progression $(\mathrm{P}=0.0198)$.

Diagnostic value of serum sIL2R level for the diagnosis of malignant lymphoma. Patients in the ML and other groups were divided into sIL2R increase-positive and -negative populations by adjusting the cutoff value of the sIL2R level $500-5,000 \mathrm{U} / \mathrm{ml}$ by every 500 or $1,000 \mathrm{U} / \mathrm{ml}$ (Table IV). The Sn decreased from 47.4 to $15 \%$ by incrementing the point of the cutoff value, whereas the Sp increased from 76.5 to $100 \%$. When the Sp was $80 \%$, the threshold level of sIL2R was $1,104 \mathrm{U} / \mathrm{ml}$, at which point ML was suspected. When the cutoff value was increased up to $1,500 \mathrm{U} / \mathrm{ml}$, the $\mathrm{Sp}$, odds ratio, and positive likelihood ratio (LR+) were elevated to $87 \%, 4.28$, and 2.997 , respectively. Even when the cutoff value was adjusted to $2,000 \mathrm{U} / \mathrm{ml}$, the $\mathrm{Sp}$, odds ratio and $\mathrm{LR}+$ were elevated to 93.2\%, 17.07 and 5.058, respectively.

The ROC curve for prediction of lymphoma by SIL2R is shown in Fig. 2. The area under the curve (AUC) was 0.695. The curve was nearest to the left corner of the plot when the threshold was $1,946 \mathrm{U} / \mathrm{ml}$, at which point the $\mathrm{Sn}$ and $\mathrm{Sp}$ were 34.6 and $93.2 \%$, respectively. The positive predictive value (PPV) and negative predictive value (NPV) at this threshold were 85.2 and $55.6 \%$, respectively. This threshold was considered appropriate, as the Sp declined rapidly when a threshold of $<1,946$ was applied. Age and LDH levels appeared to contribute to the diagnosis of ML. By contrast, WBC and CRP did not appear to be predictive of the presence of ML. The ROC curve was nearest to the left corner of the plot when the thresholds were as follows: Age 46 years, LDH $173 \mathrm{U} / 1$, and sIL2R 1,946 U/ml (Fig. 2).

Risk factors in the diagnosis of malignant lymphoma by multivariate analysis. The multivariate analysis of the serum SIL2R level and several factors (age, sex, presence of fever, WBC count, LDH and sIL2R) was performed using the multivariate logistic regression model. The AUC was 0.695 for sIL2R alone, and increased to 0.733 by including age $>46$ years an LDH $>173 \mathrm{U} / 1$ with the sIL2R cutoff value at 1,946 U/ml (Fig. 3). The adjusted odds ratio of the sIL2R level was 5.97.

This analysis suggests that the risk factors for the diagnosis of $\mathrm{ML}$ are age $>46$ years, $\mathrm{LDH}>173 \mathrm{U} / 1$, as well as sIL2R 
A

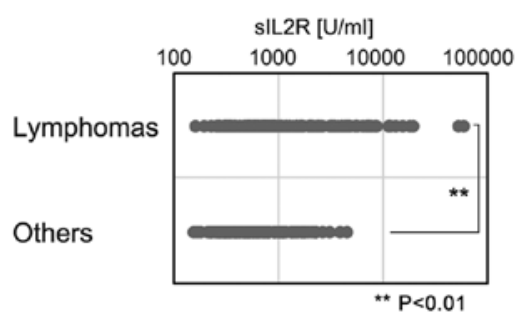

C

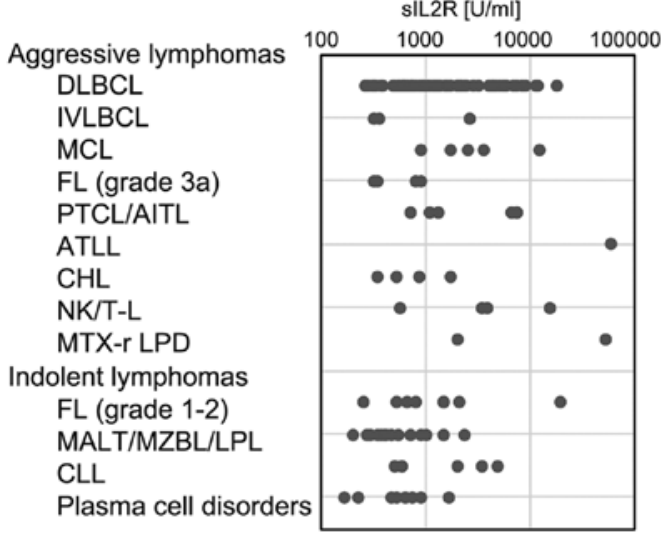

$\mathrm{E}$

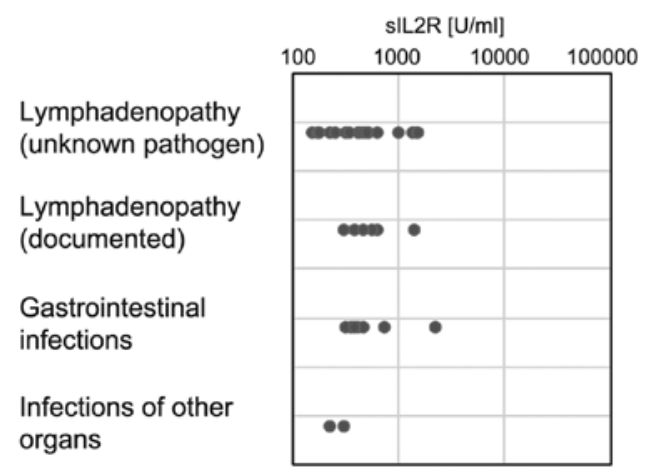

B

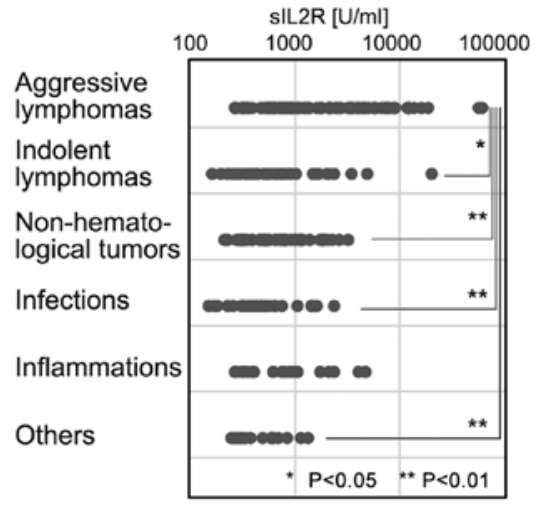

D

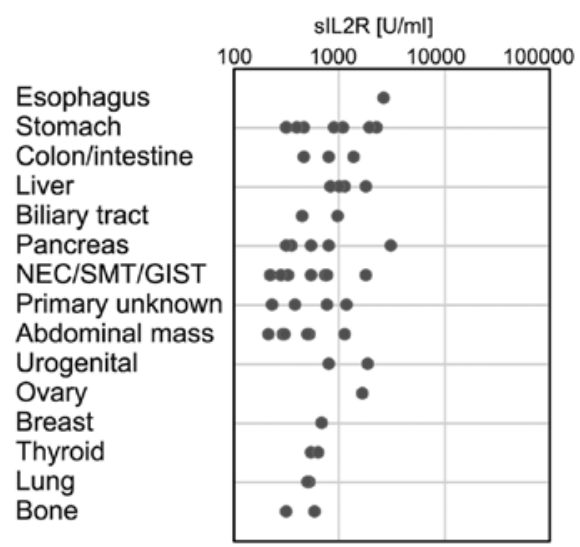

$\mathrm{F}$

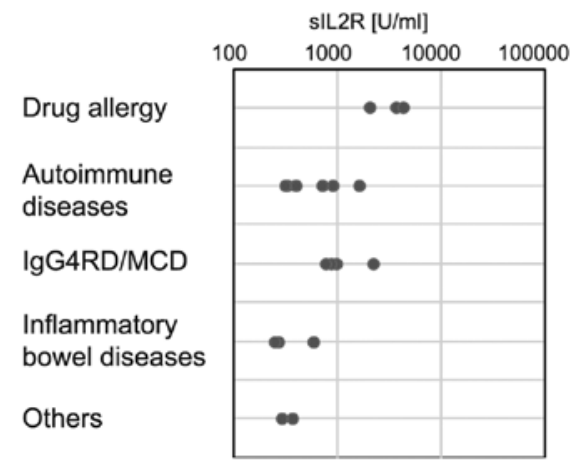

Figure 1. sIL2R levels in each category and subcategory. Dot plot of serum sIL2R levels in each diagnosis category. (A) Comparison of the serum sIL2R levels in the lymphoma group and the non-lymphoid group. (B) Serum sIL2R levels for each disease category, including aggressive or indolent types of lymphomas, CLL, plasma cell disorders including plasma cell myeloma, infection, non-infectious inflammation, and others. (C) Serum sIL2R levels of each of the detailed subtypes of aggressive lymphomas, indolent lymphomas, CLL and plasma cell disorders, including plasma cell myeloma. (D) Serum sIL2R levels in various non-lymphoid tumors. (E) Serum sIL2R levels in various infectious diseases. (F) Serum sIL2R levels in various inflammatory diseases. sIL2R, soluble interleukin-2 receptor; CLL, chronic lymphocytic leukemia; DLBCL, diffuse large B-cell lymphoma; IVLBCL, intravascular diffuse large B-cell lymphoma; MCL, mantle cell lymphoma; FL, follicular lymphoma; AITL, angioimmunoblastic T-cell lymphoma; PTCL, peripheral T-cell lymphoma; ATLL, adult T-cell leukemia/lymphoma; CHL, classical Hodgkin's lymphoma; NK/T-L, natural killer/T-cell lymphoma; MTX-r LPD, methotrexate-related lymphoproliferative disorders; MALT, extranodal mucosa-associated lymphoid tissue type lymphoma; MZBL, nodal marginal zone B-cell lymphoma; LPL, lympho-plasmacytic lymphoma; NEC, neuroendocrine carcinoma; SMT, submucosal tumor; GIST, gastrointestinal stromal tumor; IgG4-RD, IgG4-related disease; MCD, multicentric Castleman's disease.

$>1,946 \mathrm{U} / \mathrm{ml}$. However, the diagnostic value of higher age [Sn 0.17, Sp 0.287, PPV 0.598, NPV 0.750, accuracy 0.625, LR+ 1.286, negative likelihood ratio (LR-) 0.288] and LDH (Sn 0.767, Sp 0.452, PPV 0.617, NPV 0.627, accuracy 0.621, LR+ 1.400, LR- 0.515) was relatively small compared with that of higher levels of sIL2R (Sn 0.346, Sp 0.930, PPV 0.852, NPV 0.552, accuracy 0.617, LR+ 4.972, LR- 0.703). When these factors were combined with sIL2R, the diagnostic value mildly improved (Sn 0.293, Sp 0.957, PPV 0.886, NPV 0.539, accuracy 0.601, LR+ 6.744, LR- 0.739) compared with that of sIL2R alone (Table V).

In summary, sIL2R appears to be a more useful predictive marker compared with any other clinical parameters for the diagnosis of lymphomas. 
Table IV. Diagnostic characteristic in each cut-off level of sIL2R for the diagnosis of ML.

\begin{tabular}{lccccccccc}
\hline Cut-off sIL2R level (U/ml) & ML/other & $\mathrm{Sn}$ & $\mathrm{Sp}$ & $\mathrm{PPV}$ & $\mathrm{NPV}$ & Accuracy & LR+ & LR- & OR \\
\hline$\geq 500$ & $105 / 61$ & 0.79 & 0.47 & 0.63 & 0.66 & 0.64 & 1.4 & 0.45 & 3.32 \\
$<500$ & $28 / 54$ & & & & & & & & \\
$\geq 1,000$ & $63 / 27$ & 0.47 & 0.77 & 0.70 & 0.56 & 0.61 & 2.02 & 0.69 & 2.93 \\
$<1,000$ & $70 / 88$ & & & & & & & & \\
$\geq 1,500$ & $52 / 15$ & 0.39 & 0.87 & 0.78 & 0.55 & 0.61 & 3.00 & 0.70 & 4.28 \\
$<1,500$ & $81 / 100$ & & & & & & & & \\
$\geq 2,000$ & $46 / 8$ & 0.35 & 0.93 & 0.85 & 0.55 & 0.62 & 4.97 & 0.70 & 7.07 \\
$<2,000$ & $87 / 107$ & & & & & & & & \\
$\geq 2,500$ & $37 / 4$ & 0.28 & 0.97 & 0.90 & 0.54 & 0.60 & 8.00 & 0.75 & 10.7 \\
$<2,500$ & $96 / 111$ & & & & & & & & \\
$\geq 3,000$ & $35 / 3$ & 0.26 & 0.97 & 0.92 & 0.53 & 0.59 & 10.1 & 0.76 & 13.3 \\
$<3,000$ & $98 / 112$ & & & & & & & & \\
$\geq 4,000$ & $26 / 1$ & 0.20 & 0.99 & 0.96 & 0.52 & 0.57 & 22.5 & 0.81 & 27.7 \\
$<4,000$ & $107 / 114$ & & & & & & & & \\
$\geq 5,000$ & $20 / 0$ & 0.15 & 1.00 & 1.00 & 0.50 & 0.54 & Inf & 0.85 & - \\
$<5,000$ & $113 / 115$ & & & & & & & & \\
\hline
\end{tabular}

ML, malignant lymphoma; Sn, sensitivity; Sp, specificity; PPV, positive predictive value; NPV, negative predictive value; LR+, positive likelihood ratio; LR-, negative likelihood ratio; OR, unadjusted odds ratio; Inf, infinite (could not be evaluated).

\section{Discussion}

The present study demonstrated the diagnostic characteristics of sIL2R in the diagnosis of lymphomas. There have been few reports evaluating the value of serum sIL2R levels for the differential diagnosis of lymphoma from other conditions in the clinical setting.

Nakase et al (7) reported that the sIL2R levels are elevated in patients with hematological neoplasms and non-hematological solid tumors compared with those in healthy subjects. Furthermore, extremely high levels $(>3,000 \mathrm{U} / \mathrm{ml})$ are only observed in acute leukemia or malignant lymphomas. These findings suggest that sIL2R levels may be a useful marker for the differential diagnosis between hematological neoplasms and non-hematological solid tumors in patients with bulky diseases (7). Patients with infectious diseases were excluded from this study. Similar results were observed in pediatric patients with leukemia, lymphoma and malignant solid tumors (3). As lymphoma may mimic a wide variety of diseases, the differential diagnosis of infectious or inflammatory diseases is crucial in patients presenting with inflammatory symptoms.

Tsujioka et al retrospectively analyzed sIL2R levels in consecutive ML patients and compared them with those in non-hematological diseases categorized by initial diagnosis (8). They compared the sIL2R levels between patients with ML and patients in the control group, which was divided into 6 categories as follows: Autoimmune diseases, non-hematological tumors, infections, fever of unknown origin (FUO), lymphadenopathy, and others. They observed that sIL2R was moderately increased in non-hematological diseases, with higher levels at 1,500 U/ml, at which level the PPV and LR+ were also elevated, indicating diagnostic accuracy for lymphomas.
In the present study, control groups were categorized by final diagnosis according to the observed clinical outcome. Our data also suggest that the diagnostic threshold of sIL2R, in which ML is considered, is at 1,104-1,500 U/ml, at which point $\mathrm{Sp}$ is elevated to $80 \%$. In our cohort, the optimal cutoff level of sIL2R for the diagnosis of lymphoma was 1,946-2,000 U/ml, at which point the $\mathrm{Sn}$ was $35 \%$ and the $\mathrm{Sp}$ was $93 \%$, strongly suggesting the diagnosis of ML. At those levels, serum SIL2R appears to be more useful compared with any other non-invasive marker for the diagnosis of lymphomas.

In the present study, aggressive lymphomas exhibited the highest serum sIL2R levels; however, indolent lymphomas exhibited a mild elevation, similar to other disease categories. Furthermore, among aggressive lymphomas, the sIL2R levels in patients with T/NK lymphomas (even when ATLL was excluded) were significantly higher compared with those in patients with aggressive B-cell lymphomas. These findings are consistent with previous reports (6-8).

In Japan, serum sIL2R measurement has been introduced in clinical practice $(1,4,6-8,10-12)$ and has been commercially available and covered by insurance since October 1994, for the purpose of evaluating the response after treatment and disease monitoring after remission in patients with ATLL and non-Hodgkin lymphomas. Furthermore, from April 2006 onwards, sIL2R has been utilized for screening as a tumor marker when there is suspected ATLL or non-Hodgkin lymphoma. At present, SIL2R is often used for patients with suspected lymphomas for screenings and differential diagnosis of tumors with uncommon presentation, lymphadenopathy with atypical course, cases with FUO, or even unidentified complaints, as lymphoma may present with various clinical symptoms, mimicking a number of conditions (13), and may be considered in the differential diagnosis of a wide variety of diseases. 

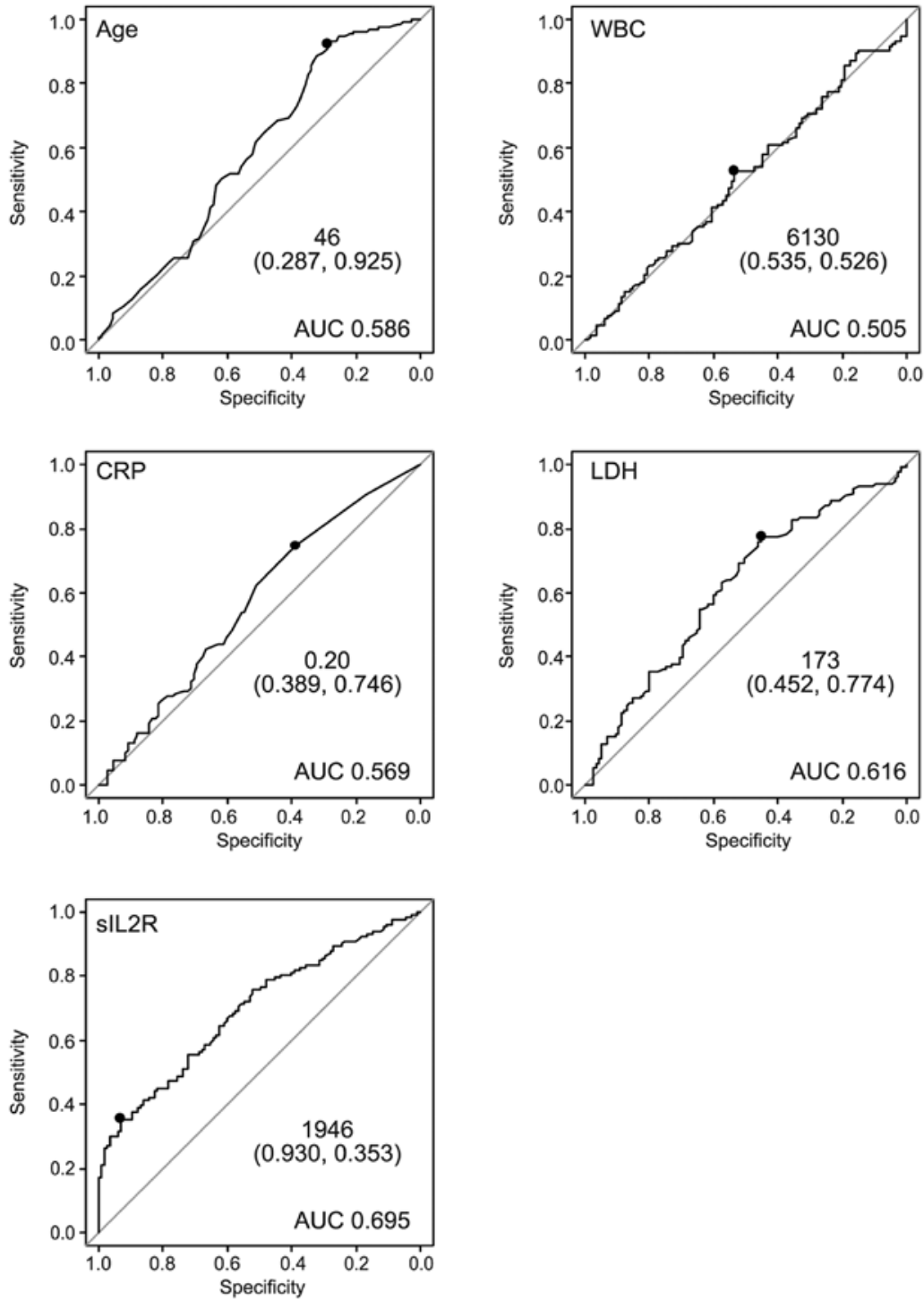

Figure 2. The ROC curve for prediction of lymphoma by several clinical parameters. The ROC curve for prediction of lymphoma by several clinical parameters (age, WBC count, CRP, LDH, sIL2R). WBC and CRP did not appear to contribute to the diagnosis of ML. By contrast, age, LDH, and sIL2R appeared to be of diagnostic value for the presence of ML. ROC, receiver operating characteristic; WBC, white blood cell; CRP, C-reactive protein; LDH, lactate dehydrogenase; sIL2R, soluble interleukin-2 receptor; AUC, area under the ROC curve; ML, malignant lymphoma.

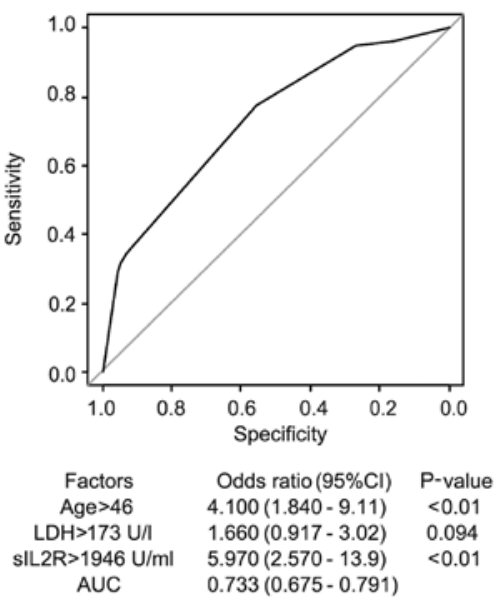

Figure 3. Multivariate logistic regression model: Risk factors for malignant lymphoma. The AUC of the sIL2R level was elevated to 0.733 by including participants aged $>46$ years and LDH levels of $>173 \mathrm{U} / 1$ to the sIL2R cut-off value at $1,946 \mathrm{U} / \mathrm{ml}$. AUC, area under the receiver operating characteristic curve; sIL2R, soluble interleukin-2 receptor; LDH, lactate dehydrogenase.
It appears difficult to distinguish ML from other conditions. However, the present study reported some evocative findings. Extremely high sIL2R levels strongly suggest the diagnosis of aggressive lymphoma, although common false-positive exceptions should be considered, including advanced non-lymphoid solid tumors with splenic involvement, drug allergies presenting with marked inflammatory symptoms, and tuberculosis or other infectious diseases. In addition, some common false-negative cases include indolent lymphomas or patients with low tumor burden.

We herein attempted to evaluate sIL2R as a tumor marker for screening and differential diagnosis using data collected from consecutive patients with suspected lymphoma who had their sIL2R levels measured in various clinical presentations. Further investigations are required to evaluate the usefulness of sIL2R in specific clinical settings to clearly determine the subcategory of patients with clinical symptoms or characteristics for whom sIL2R measurement may prove useful. For example, sIL2R was found to be useful as a diagnostic 
Table V. Diagnostic characteristic in each risk group for the diagnosis of ML.

\begin{tabular}{|c|c|c|c|c|c|c|c|c|}
\hline Risk factors & ML/other & $\mathrm{Sn}$ & $\mathrm{Sp}$ & PPV & NPV & Accuracy & LR+ & LR- \\
\hline \multicolumn{9}{|l|}{ Age, years } \\
\hline$>46$ & $122 / 82$ & 0.92 & 0.29 & 0.60 & 0.75 & 0.63 & 1.29 & 0.29 \\
\hline$\leq 46$ & $11 / 33$ & & & & & & & \\
\hline \multicolumn{9}{|l|}{ LDH, U/1 } \\
\hline$>173$ & $102 / 63$ & 0.77 & 0.45 & 0.62 & 0.63 & 0.62 & 1.40 & 0.52 \\
\hline$\leq 173$ & $31 / 52$ & & & & & & & \\
\hline \multicolumn{9}{|l|}{ sIL2R, U/ml } \\
\hline$>1,946$ & $46 / 8$ & 0.35 & 0.93 & 0.85 & 0.55 & 0.62 & 4.97 & 0.70 \\
\hline$\leq 1,946$ & $87 / 107$ & & & & & & & \\
\hline \multicolumn{9}{|c|}{ Number of risk factors } \\
\hline 0 & $\begin{array}{c}5 / 18 \\
128 / 97\end{array}$ & 0.04 & 0.84 & 0.22 & 0.43 & 0.41 & 0.24 & 1.14 \\
\hline 1 & $\begin{array}{c}25 / 46 \\
108 / 69\end{array}$ & 0.19 & 0.60 & 0.35 & 0.39 & 0.38 & 0.47 & 1.35 \\
\hline 2 & $\begin{array}{l}64 / 46 \\
69 / 69\end{array}$ & 0.48 & 0.60 & 0.58 & 0.50 & 0.54 & 1.20 & 0.87 \\
\hline 3 & $\begin{array}{c}39 / 5 \\
94 / 110\end{array}$ & 0.29 & 0.96 & 0.89 & 0.54 & 0.60 & 6.74 & 0.74 \\
\hline
\end{tabular}

ML, malignant lymphoma; Sn, sensitivity; Sp, specificity; PPV, positive predictive value; NPV, negative predictive value; LR+, positive likelihood ratio; LR-, negative likelihood ratio.

marker in hemophagocytic syndromes/hemophagocytic lymphohistiocytosis associated with lymphomas (14-16).

In conclusion, as serum sIL2R levels may be elevated in several pathological conditions, including inflammatory or neoplastic diseases, they appear to be a less specific marker. However, the findings of the present study indicate that higher levels of sIL2R strongly suggest the presence of lymphoma, and may thus be useful for the diagnosis of aggressive types of lymphomas, after ruling out other false-positive conditions.

\section{Acknowledgements}

Not applicable.

\section{Funding}

No funding was received.

\section{Availability of data and materials}

The datasets used and/or analyzed during the present study are available from the corresponding author on reasonable request.

\section{Authors' contributions}

JM and HM designed the study and wrote the initial draft of the manuscript. KA contributed to refining the figures. KA, AW, IY and TS contributed to the acquisition, analysis and interpretation of data. $\mathrm{HO}$ and MK verified the analytical methods. All authors discussed the results and contributed to the final manuscript.

\section{Ethics approval and consent to participate}

The study obtained ethical approval for the use of an opt-out methodology due to the low risk to the patient and the potential benefit for the patient of adequate diagnosis based on unbiased information.

\section{Patient consent for publication}

Not applicable.

\section{Competing interests}

The authors declare that they have no competing interests.

\section{References}

1. Yasuda N, Takamatsu T, Kanoh T and Uchino H: Serum levels of soluble interleukin 2 receptor in patients with non-haematological disorders. Br J Haematol 69: 573, 1988.

2. Rubin LA, Galli F, Greene WC, Nelson DL and Jay G: The molecular basis for the generation of the human soluble interleukin 2 receptor. Cytokine 2: 330-336, 1990.

3. Bien E and Balcerska A: Serum soluble interleukin 2 receptor alpha in human cancer of adults and children: A review. Biomarkers 13: 1-26, 2008.

4. Yasuda N, Lai PK, Ip SH, Kung PC, Hinuma Y, Matsuoka M, Hattori T, Takatsuki K and Purtilo DT: Soluble interleukin 2 receptors in sera of Japanese patients with adult $\mathrm{T}$ cell leukemia mark activity of disease. Blood 71: 1021-1026, 1988.

5. Ambrosetti A, Nadali G, Vinante F, Ricetti MM, Todeschini G, Morosato L, de Sabata D, Bergamo Andreis IA, Chilosi M, Semenzato G, et al: Soluble interleukin-2 receptor in hairy-cell leukemia: A reliable marker of disease. Int J Clin Lab Res 23: 34-37, 1993. 
6. Yoshida N, Oda M, Kuroda Y, Katayama Y, Okikawa Y, Masunari T, Fujiwara M, Nishisaka T, Sasaki N, Sadahira Y, et al: Clinical significance of sIL-2R levels in B-cell lymphomas. PLoS One 8: e78730, 2013.

7. Nakase K, Tsuji K, Tamaki S, Tanigawa M,Ikeda T, Miyanishi E and Shiku H: Elevated levels of soluble interleukin-2 receptor in serum of patients with hematological or non-hematological malignancies. Cancer Detect Prev 29: 256-259, 2005.

8. Tsujioka T, Kishimoto M, Kondo T, Matsuoka A, Tasaka T, Sugihara T, Wada $\mathrm{H}$ and Tohyama $\mathrm{K}$ : The impact of serum soluble interleukin-2 receptor levels on the diagnosis of malignant lymphoma. Kawasaki Med J 37: 19-27, 2011.

9. Kanda Y: Investigation of the freely available easy-to-use software 'EZR' for medical statistics. Bone Marrow Transplant 48: 452-458, 2013

10. Kamihira S, Atogami S, Sohda H, Momita S, Yamada Y and Tomonaga M: Significance of soluble interleukin-2 receptor levels for evaluation of the progression of adult T-cell leukemia. Cancer 73: 2753-2758, 1994.

11. Murakami S, Satomi A, Ishida K, Murai H, Matsuki M and Hashimoto T: Serum-soluble interleukin-2 receptor concentrations in patients with gastric cancer. Cancer 74: 2745-2748, 1994.
12. Goto $H$, Tsurumi H, Takemura M, Ino-Shimomura $Y$, Kasahara S, Sawada M, Yamada T, Hara T, Fukuno K, Goto N, et al: Serum-soluble interleukin-2 receptor (sIL-2R) level determines clinical outcome in patients with aggressive non-Hodgkin's lymphoma: In combination with the International Prognostic Index. J Cancer Res Clin Oncol 131: 73-79, 2005.

13. Nakashima MO, Roy DB, Nagamine M, Roullet MR, Gabriel CA, Sood SL and Bagg A: Intravascular large B-cell lymphoma: A mimicker of many maladies and a difficult and often delayed diagnosis. J Clin Oncol 29: e138-e140, 2011.

14. Tabata $C$ and Tabata R: Possible prediction of underlying lymphoma by high sIL-2R/ferritin ratio in hemophagocytic syndrome. Ann Hematol 91: 63-71, 2012.

15. Hayden A, Lin M, Park S, Pudek M, Schneider M, Jordan MB, Mattman A and Chen LYC: Soluble interleukin-2 receptor is a sensitive diagnostic test in adult HLH. Blood Adv 1: 2529-2534, 2017.

16. Lin M, Park S, Hayden A, Giustini D, Trinkaus M, Pudek M, Mattman A, Schneider M and Chen LYC: Clinical utility of soluble interleukin-2 receptor in hemophagocytic syndromes: A systematic scoping review. Ann Hematol 96: 1241-1251, 2017. Attribution 4.0 International (CC BY 4.0) License. 